Open Access

\title{
Intraosseous intraneural perineurioma derived from the inferior alveolar nerve with an abnormality of chromosome 22 and expression of the BCR-ABL fusion gene: report of a case and review of recent literature
}

Jun Kurihara ${ }^{1 *}$ D, Satoshi Yokoo ${ }^{1}$, Miku Ichikawa ${ }^{1}$, Takahiro Shimizu' ${ }^{1}$ Masaru Ogawa ${ }^{1}$ and Mai Seki ${ }^{1,2}$

\begin{abstract}
Background: Perineurioma (PN) is a peripheral nerve disease that primarily develops in the limbs and trunk and very rarely occurs in the oral cavity. PN is classified into two types: intraneural perineurioma (INPN) and soft tissue perineurioma (extraneural perineurioma, ENPN). In this article, we report a patient with mandibular body INPN derived from the perineurium of the inferior alveolar nerve.

Case presentation: The patient was a 43-year-old male. He consulted our department for a detailed examination of the right mandibular body. A biopsy was performed at another hospital and he was diagnosed with a schwannoma. At his first visit, hypesthesia extending from the right lower lip to the mental region was recognized and enlargement of the right mandibular canal was confirmed with X-ray CT and MRI. Considering the possibility of future tumor growth, we extirpated the tumor under general anesthesia. Cystic tumor was seen continuously in the inferior alveolar nerve. Immunohistologically, the tumor cells were positive for Glut-1, weakly positive for EMA, and weakly positive for Claudin-1, and the histopathological diagnosis was INPN. In addition, absence of the BCR region of chromosome 22 and expression of the BCR-ABL fusion gene were observed by fluorescent in situ hybridization (FISH), and a chromosome 22 abnormality was confirmed. These findings indicated that the disease was a neoplastic lesion.
\end{abstract}

Conclusion: Expression of the BCR-ABL fusion gene in INPN that develops in the oral cavity is thought to be very rare, and to the best of our knowledge, ours is the first case to be reported in the literature. About three postoperative years have passed, but findings suggestive of recurrence have not been observed.

Keywords: Perineurioma, Chromosome 22 abnormality, BCR-ABL fusion gene, INPN, ENPN

\footnotetext{
* Correspondence: jkurihara@gunma-u.ac.jp

${ }^{1}$ Department of Oral and Maxillofacial Surgery, and Plastic Surgery, Gunma

University Graduate School of Medicine, 4-39-15 Showa-machi, Maebashi,

Gunma 371-8511, Japan

Full list of author information is available at the end of the article
}

(c) The Author(s). 2018 Open Access This article is distributed under the terms of the Creative Commons Attribution 4.0 International License (http://creativecommons.org/licenses/by/4.0/), which permits unrestricted use, distribution, and 


\section{Background}

Perineurioma $(\mathrm{PN})$ is a peripheral nerve disease that primarily develops in the limbs and trunk. Development in the oral cavity is rare. $\mathrm{PN}$ is classified into two types: intraneural perineurioma (INPN) and soft tissue perineurioma (extraneural perineurioma, ENPN). The incidence of INPN is lower than that of ENPN. A search of PubMed from 1981 until 2017 revealed only four case reports on mandibular INPN, including our report [13]. In addition, to the best of our knowledge, this is the first report on INPN of the inferior alveolar nerve with a chromosome 22 abnormality and BCR-ABL fusion gene expression, simultaneously. In this article, we report a patient with INPN in the mandibular body region derived from the perineurium of the inferior alveolar nerve and review the literature to analyze the pathogenesis.

\section{Case presentation}

A 43-years-old male of Japanese Brazilian with hypesthesia from the right lower lip to the mental region consulted a hospital for the first visit. Computed tomography $(\mathrm{CT})$ revealed a round radiolucent area in the right mandibular body, and biopsy was carried out, leading to a diagnosis of schwannoma. He was referred to the Department of Oral and Maxillofacial Surgery, Gunma University Hospital, for detailed examination and treatment for the first time in March 2015.

His physical status was moderate and nutritional status favorable. There was no other notable factor. On visual inspection, there was no tumor lesion in the oral cavity (Fig. 1). Hypesthesia extending from the right lower lip to the mental region was noted, and the perception level was approximately $50 \%$ of that on the unaffected side. CT imaging revealed dilation of the mandibular canal (Fig. 2), and magnetic resonance imaging (MRI) showed an irregular high signal intensity on horizontal sections of short inversion time inversion recovery (STIR). On

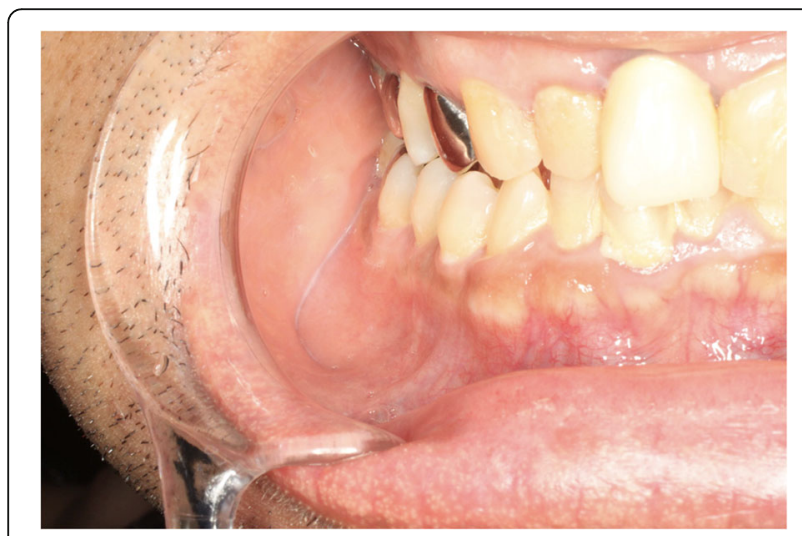

Fig. 1 Intraoral findings. Although no obvious neoplastic lesions were observed, hypesthesia extending from the right lower lip to the mental region was noted sagittal sections, dilation of the right mandibular canal was observed around the mandibular foramen. Dynamic images showed crescendo enhancement (Fig. 3).

Preoperative biopsy findings carried out at the first hospital visited by the patient suggested a schwannoma involving the Antoni A region. Briefly, the lesion was immature, suggesting the presence of an active potential. Considering the risk of future tumor growth, the tumor was extirpated under general anesthesia in late April 2015. A macroscopically observed intraoperative finding was a cystic tumor $(3.2 \times 1.0 \mathrm{~cm})$ associated with the inferior alveolar nerve and vessels; hence, the inferior alveolar nerve was ligated/cut and extirpated as a mass (Fig. 4a, b). Intraoperative rapid diagnosis confirmed the absence of tumor cells at the margins of the resected nerve specimen. During the 2-year postoperative follow-up, radiopacity was enhanced at the wound site after extirpation of the tumor, confirming favorable bone outgrowth (Fig. 4c, d). There have been no subsequent clinical findings or images suggestive of relapse.

In a hematoxylin-and-eosin ( $\mathrm{H}-\mathrm{E})$-stained specimen, bundle-like tumor proliferation was observed around the nerve fiber. In addition, outgrowth of tumor cells was noted around nerve fibers, separating the nerve fiber bundle (Fig. 5a-c).

Immunohistologically, the tumor cells were positive for Glut-1 and weakly positive for EMA and Claudin-1. The tumor cells were negative for S-100, but the residual nerve fibers were positive for S-100 (Fig. 6a-e). Based on these findings, the histopathological diagnosis was INPN. The MIB-1 expression rate of INPN, indicating its cell proliferation ability, was $1.6 \%$ (Fig. 7).

To examine the pathogenesis of this tumor, FISH, which facilitates the detection of gene localization on the chromosomes, was carried out. In this procedure, a cloned gene or DNA fragment is labeled with a non-isotopic compound and hybridized with chromosomal DNA on a glass slide to directly detect the site of in situ hybridization, as a fluorescent signal, on a chromosome. FISH is safer and simpler than conventional autoradiography. In addition, it is advantageous as the results can be obtained in a short time. As standards, the fluorescent body of chromosome 9 (orange signal) indicates the $\mathrm{ABL}$ gene, that of chromosome 22 (green signal) indicates the BCR gene, and a yellow signal indicates the BCR-ABL fusion gene. Cellular signals on INPN were visually counted and classified. Measurement criteria for detecting the ALK fusion gene using the FISH method were quoted as diagnostic criteria. The principle is similar to that of BCR-ABL fusion gene detection. Samples with $<5$ positive cells per 50 cells $(<5 /$ 50 or $<10 \%)$ were regarded as negative, and those with $>25$ positive cells per 50 cells $(>25 / 50$ or $>50 \%$ ) were regarded as positive. The results showed that signals 


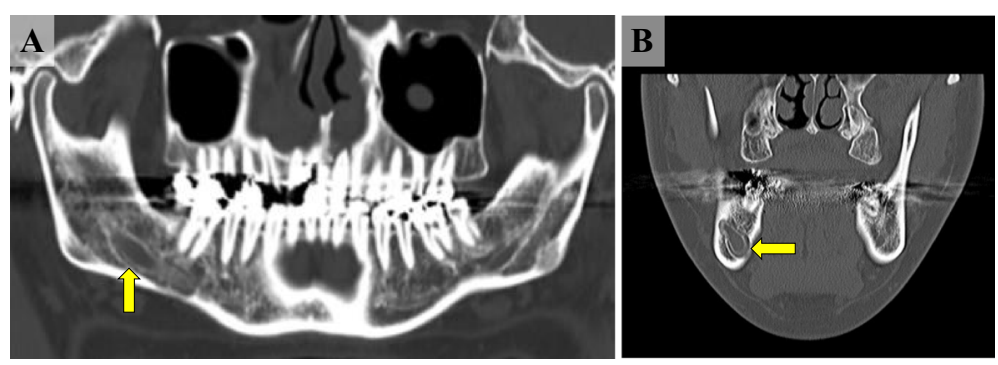

Fig. 2 X-ray findings. a X-ray CT finding (panoramic image). A radiolucent finding of a similar circle was recognized in the right mandible body (yellow arrow). $\mathbf{b}$ X-ray $C T$ finding (coronal image). We confirmed the enlargement of the right mandible canal. The destruction of the mandibular canal wall is not clear (yellow arrow)

suggestive of BCR-ABL fusion gene formation were observed in 30 of 50 cells. In 12 of the 50 cells, the green signal of the BCR gene was present in the absence of fusion gene signals, suggesting deletion of the BCR region. Thus, 42 of the 50 cells were positive, suggesting chromosome 22 abnormalities. According to the above diagnostic criteria, our patient was regarded as having a chromosome 22 abnormality. Based on this finding, it was confirmed that this disease was a tumorous lesion (Fig. 8).

\section{Discussion}

PN was first reported by Lazarus et al. in 1978 [4]. According to the WHO classification in 2013, it is defined as a "benign peripheral nerve sheath tumor consisting of perineurium cells." PN is a peripheral nerve sheath tumor, but it is rare in comparison with schwannoma or neurofibroma. PN develops in the limbs, trunk, and head and neck region of adults. Most lesions are small, measuring $\leq$ $3 \mathrm{~cm}$, although some become large. The sites of PN include the subcutaneous area, superficial layer of the dermis, and area deeper than the subcutaneous area. PN development in the oral cavity is very rare. Searching PubMed from 1981 until 2017 revealed 37 patients with oral PNs. Of these, 15 cases were INPN [2-14] including this present case (Table 1). Most reported oral INPN lesions were relatively small, and the mean diameter was approximately $2.1 \mathrm{~cm}$ $(0.75-4.0 \mathrm{~cm})$, including the present case. The most frequent site was the tongue in seven cases, next was mandibular in four including this present case, followed by the buccal mucosa in three, and lower lip in one.
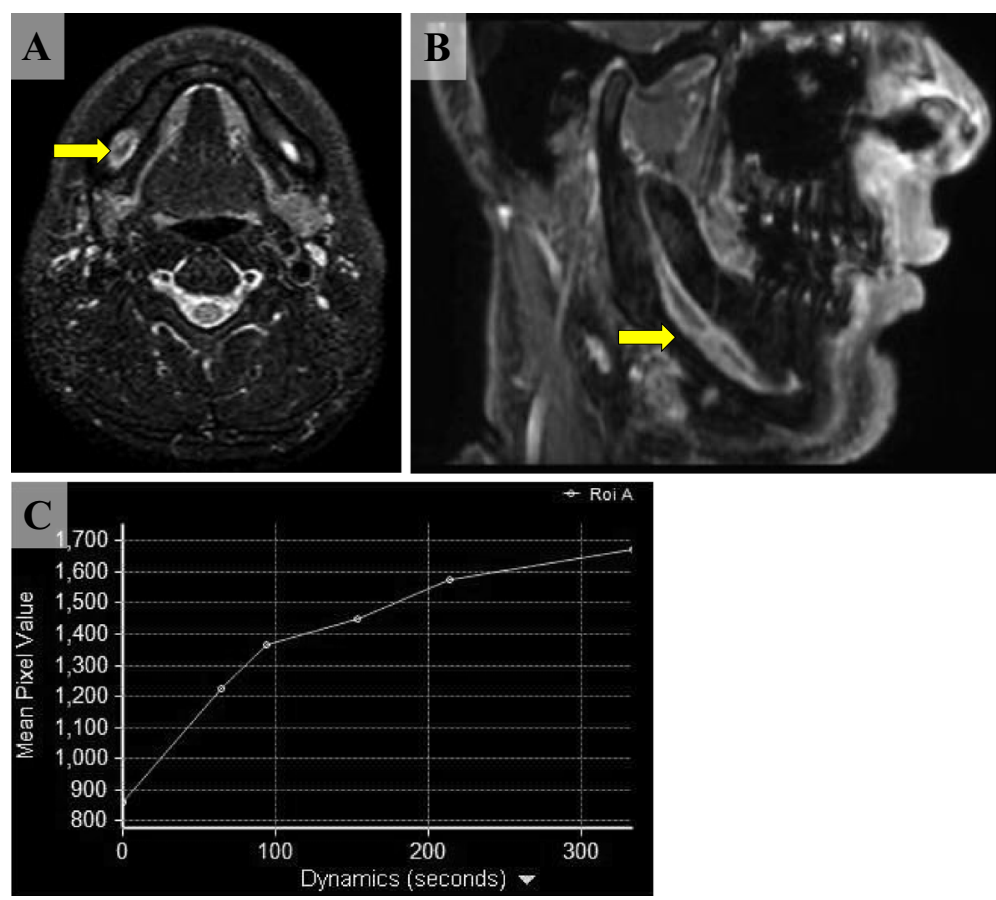

Fig. $3 \mathrm{MRI}$ findings. a Horizontal section. An irregular high signal in the STIR image was recognized (yellow arrow). b Sagittal sections. Dilation of the right mandibular canal was observed around the mandibular foramen (yellow arrow). c Dynamic images showed crescendo enhancement 

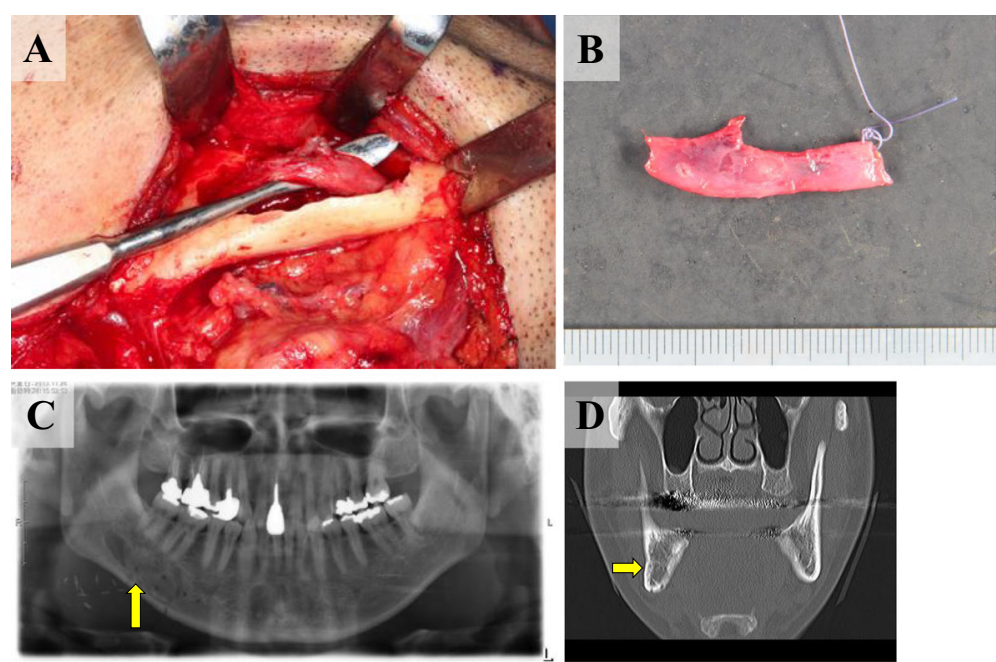

Fig. 4 a Intraoperative findings. A cystic tumor of $3.2 \times 1.0 \mathrm{~cm}$ was observed macroscopically following the inferior alveolar nerve. $\mathbf{b}$ Extirpation of tumor findings. The surrounding inferior alveolar neurovascular bundle was ligated and cut and removed as a lump. c, d Postoperative panoramic X-ray and X-ray CT findings. The radiopacity of the excised cavity was enhanced, and hyperostosis was confirmed

INPN involving the sensory nerve may cause sensory disturbance, and INPN involving the motor nerve may cause dyskinesia, such as progressive muscle weakness, but it rarely induces muscular atrophy. In the present case, hypesthesia involving the right lower lip extending to the mental region was observed.

In patients with mandibular PN, it is extremely important to evaluate the required extent of inferior alveolar nerve resection during surgery, as indicated for other neurogenic tumors. For evaluation, it is necessary to remove the cortical bone around the tumor, locate the tumor directly, and examine the state of adhesion to the inferior alveolar nerve or continuity with it. In previous studies, all cases, including the present case, underwent resection, and there was no report of relapse.

\section{A}
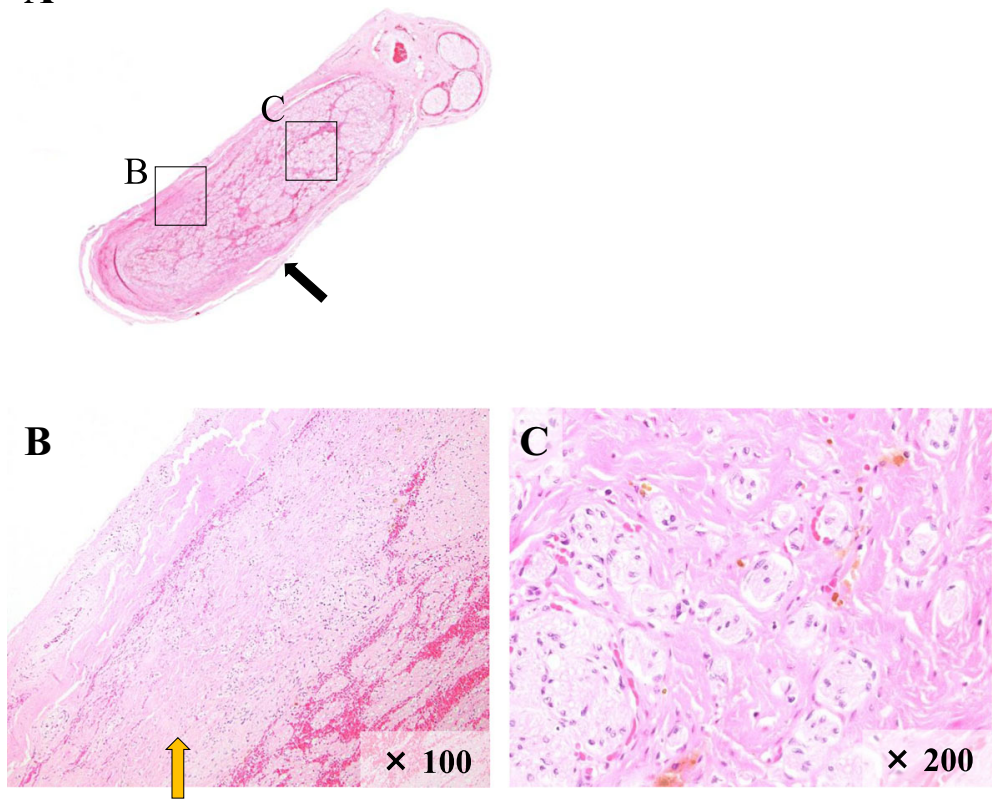

Fig. $5 \mathrm{H}$-E staining. a Weak expansion. A tumor was found in the inferior alveolar nerve and inside (black arrow). $\mathbf{b} \times 100$. A thickened perineurium cell layer was confirmed (yellow arrow). $\mathbf{c} \times 200$. A part showing a pseudo-onion bulb-like structure confirming proliferation of tumor cells was observed so as to separate the nerve fiber bundle. B and C in Fig. 5a are enlargements of the same part as Fig. 5b, c 


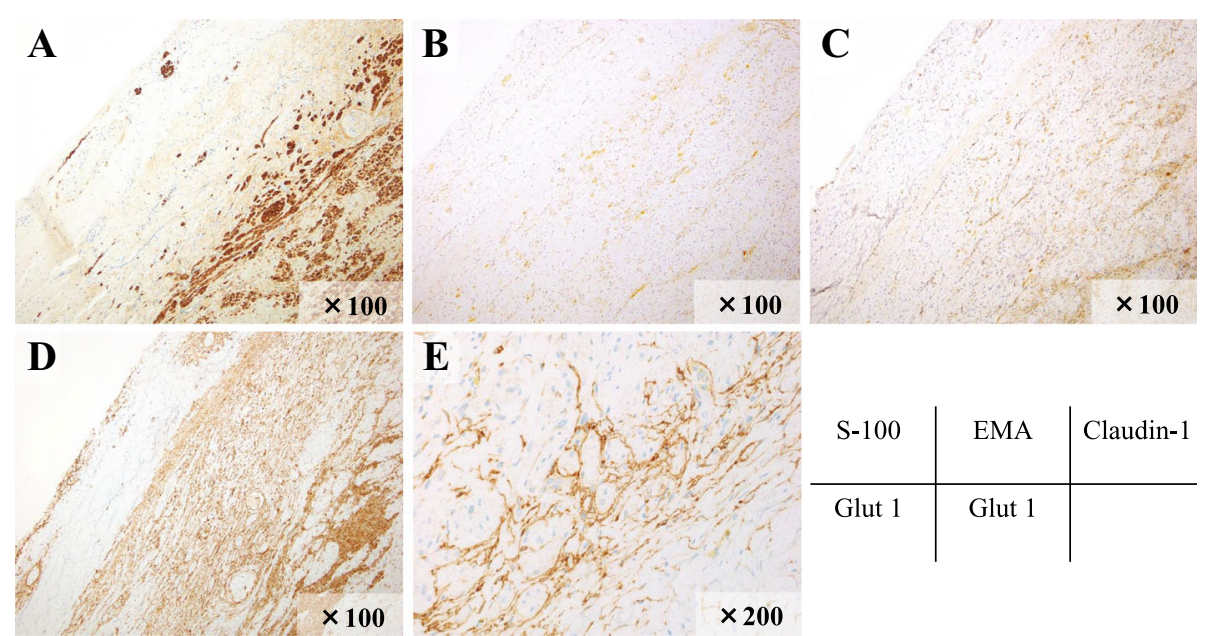

Fig. 6 Immunohistochemical staining. The tumor cells were Glut-1 positive (d, e), weakly positive EMA (b), and Claudin-1 weakly positive (c). S100 was negative for tumor cells (a), but positive for nerve fibers

INPN is a nodular lesion. A large number of pseudo-onion bulb-like structures with a scroll-like proliferation of spindle cells around axons in the inner areas of nerves comprise the tumor parenchyma. In the present case, a solid proliferation of bundle-like spindle cells containing a large amount of cell components was observed around the inferior alveolar nerve. Partially, pseudo-onion bulb-like structures, as described above, were noted. Histopathologically, INPN was suggested.

Diseases to be differentiated from PN include schwannoma and traumatic neuroma. To make a definitive diagnosis, it is important to perform PN-specific immunostaining. Representative immunostaining methods primarily used for PN diagnosis include EMA, Claudin-1, and Glut-1. EMA is a high-molecular-weight membrane glycoprotein of epithelial cells. Patients with

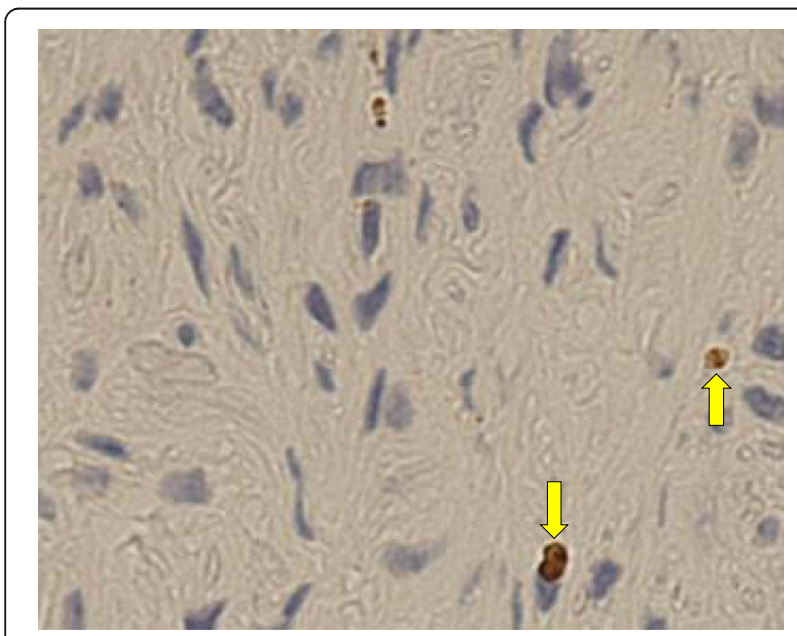

Fig. 7 Expression rate of MIB-1 (Ki-67). The expression rate (\%) of MIB-1 in INPN was 1.6\% (positive nucleus: yellow arrow) meningioma or peripheral nerve sheath tumors derived from perineurium cells show positive reactions to EMA. Claudin-1 is a tight-junction component protein, and it is utilized as a marker of the perineurium or its differentiation. Glut-1 is a member of the 12-pass transmembrane membrane protein family and is used as a perineurium cell marker. On the other hand, neuroglial and Schwann cells are positive for S-100 protein and negative for PN (Table 2). In the present case, tumor cells were positive for Glut-1/EMA and weakly positive for Claudin-1 on immunohistochemical staining. There were no S-100 protein-positive cells. Furthermore, H-E staining confirmed characteristic findings, leading to a definitive diagnosis of a perineurium cell-derived peripheral nerve sheath tumor, that is, INPN.

Concerning the pathogenesis of PN, previous studies considered that localized hypertrophic neuropathy of perineurium cells in response to trauma, inflammation, and ischemia might be involved. However, recent studies reported the partial deletion of chromosome 22 (22q11) and chromosome 22 abnormalities [15, 16]. The hypothesis that PN may be a tumorous disease has been strongly supported, but whether it is a true neoplastic or reactive lesion has remained controversial. Previous studies reported the frequent expression of p53 and specific gene abnormalities in patients with PN. On the other hand, there were few phenomena, such as trauma or ischemia, in previously reported patients with PN despite the hypothesis that PN may be a reactive lesion. In recent years, it has been reported that chromosome 22 abnormality leading to the alteration of tumor suppressor genes that cause clonal proliferation of perineurial cells is a pathogenesis of its development in PN. In other words, cytogenetic changes and chromosomal abnormalities supporting the fact that $\mathrm{PN}$ is not a reactive 

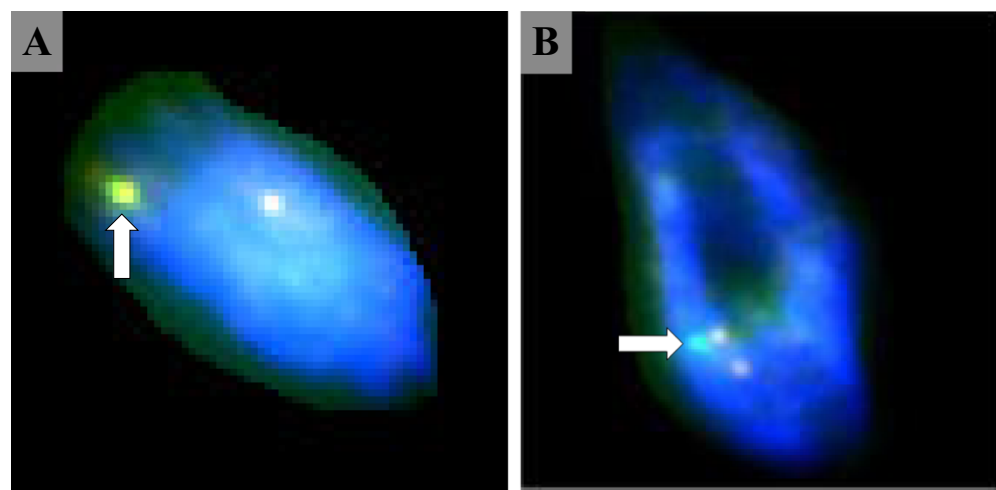

Fig. 8 FISH (fluorescent in situ hybridization) method. a The BCR-ABL fusion gene shows a yellow signal. A yellow signal was observed, and expression of BCR-ABL fusion gene was confirmed (white arrow). $\mathbf{b}$ An orange signal indicates $A B L$ gene and a green signal indicates $B C R$ gene. Cells with one green signal of BCR gene were observed, and lack of BCR region was confirmed (white arrow)

change but a true benign tumor are thought to be the etiology [14].

Assuming that PN could be a tumorous disease, it is important to confirm the expression of cell growth markers. Proliferative cell nuclear antigen (PCNA), which is a nuclear protein that secondarily promotes DNA replication, and MIB-1 (Ki-67), which is a cell marker during cell growth in the G1, S, G2, and M phases of the cell cycle, were represented. The MIB-1 expression rate with $\mathrm{PN}$ involving the trunk ranges from approximately 4 to $14 \%$ [15]. However, based on the results of this survey, MIB-1 expression was detected in two patients with PN involving the oral and maxillofacial region, including the present case [17]. Accounting for $\leq 5 \%$, the PCNA expression rate was $16.8 \%$ and the
MIB-1 expression rate was $1.6 \%$ in the present case, suggesting proliferative capacity. This was within the range of expression rates in benign tumors. This activity is low, and the risk of relapse may be low if an adequate extent of resection is established.

FISH studies may clarify the process of tumorous proliferation related to $\mathrm{PN}$-specific gene abnormalities. Previous studies confirmed the $22 \mathrm{q} 11$ deletion as a chromosome abnormality in patients with benign or malignant schwannoma, neurofibroma, or meningioma. Chromosome 22 deletion was confirmed in patients with peripheral nerve sheath tumors $[15,18,19]$, and the long arm of chromosome 22 is known to involve a tumor suppressor gene, which may be involved in the pathogenesis of these diseases. Therefore, if the partial deletion

Table 1 Clinical characteristics of oral intraneural perineuriomas (INPN)

\begin{tabular}{|c|c|c|c|c|c|c|c|}
\hline Authors/year & Patient no. & Age & Sex & Location & Size $(\mathrm{cm})$ & Treatment & Follow-up \\
\hline Damm et al. 2003 [5] & 1 & 26 & $\mathrm{~F}$ & Tongue & 0.75 & Resection & ND \\
\hline Huguet et al. 2004 [1] & 2 & 64 & M & Mandible & 2.0 & Resection & ND \\
\hline Da Cruz Perez et al. 2006 [6] & 3 & 12 & M & Tongue & 0.6 & Resection & $6 \mathrm{~m} \mathrm{NR}$ \\
\hline Siponen et al. 2007 [7] & 4 & 18 & $F$ & Buccal mucosa & 2.0 & Resection & $2 y N R$ \\
\hline Boyanton et al. 2007 [8] & 5 & 6 & $F$ & Tongue & 0.8 & Resection & $4 \mathrm{~m} \mathrm{NR}$ \\
\hline Dundr et al. 2007 [9] & 6 & 16 & M & Buccal mucosa & 1.5 & Resection & $2 y \mathrm{NR}$ \\
\hline Kang et al. 2007 [10] & 7 & 16 & M & Tongue & 10 & Resection & $6 \mathrm{~m} \mathrm{NR}$ \\
\hline Tanaka et al. 2009 [11] & 8 & 34 & $\mathrm{~F}$ & Tongue & 0.6 & Resection & $8 \mathrm{~m} \mathrm{NR}$ \\
\hline Vencio et al. 2009 [2] & 9 & 59 & $F$ & Mandible & $\mathrm{N} / \mathrm{A}$ & Resection & 3y NR \\
\hline Rocha et al. 2009 [12] & 10 & 47 & $\mathrm{~F}$ & Tongue & 1.0 & Resection & 1y NR \\
\hline Rocha et al. 2009 [12] & 11 & 37 & M & Lower lip & 0.5 & Resection & 1y NR \\
\hline Hata et al. 2011 [3] & 12 & 42 & $\mathrm{~F}$ & Mandible & 1.8 & Resection & $6 \mathrm{~m} \mathrm{NR}$ \\
\hline Hirano et al. 2013 [13] & 13 & 14 & M & Tongue & 4.0 & Resection & $6 \mathrm{~m} \mathrm{NR}$ \\
\hline Gomes da Silva et al. 2016 [14] & 14 & 84 & M & Lower buccal fold & 2.0 & Resection & $5 y 8 m$ NR \\
\hline Present case 2018 & 15 & 43 & $M$ & Mandible & 3.2 & Resection & 3y NR \\
\hline
\end{tabular}

$M$ male, $F$ female, $N / A$ not available, ND no description, $N R$ no recurrence, $y$ year, $m$ month 
Table 2 Differential diagnosis of perineurioma (PN) in immunohistochemical staining $(\mathrm{IHC})$

\begin{tabular}{lllll}
\hline Neurogenic tumor/IHC & EMA & S-100 & Claudin-1 & Glut-1 \\
\hline Perineurioma & + & - & + & + \\
Schwannoma & - & + & - & - \\
Neurofibroma & - & + & - & - \\
Traumatic neuroma & - & + & - & - \\
\hline
\end{tabular}

+ positive, - negative, EMA epithelial membrane antigen

of chromosome 22 (deletion of the long arm) is confirmed, it can be demonstrated that $\mathrm{PN}$ is a tumorous lesion, as reported for other nerve sheath tumors, due to the loss of a tumor suppressor gene. In a recent report, there is a pseudo-perineurioma (PPN) in the oral cavity caused by the proliferation of perineurial cells in the nerve [14]. This pathogenesis is thought to be associated with reactive lesions such as traumatic stimuli or conventional fibromas. Proliferation of this PPN resembles typical histological and immunohistochemical features in INPN, and it is reported that it also presents some common findings in its clinical features of INPN. It is said that INPN occurs in major thick nerves, whereas PPN occurs in a small branch of the nerve. PPN mainly occurs in the tongue and is said to be small in size as compared with INPN $[14,20]$. However, at present, there is no clear diagnostic criterion between true $\mathrm{PN}$ and PPN. For the purpose of distinguishing, such as in this examination, cytogenetic search is considered necessary.

The BCR-ABL gene has been primarily confirmed in patients with chronic myeloid leukemia. If reciprocal translocation between chromosomes 9 and 22 occurs, a chromosome 22 that is shorter than the standard type, termed the Philadelphia $(\mathrm{Ph})$ chromosome, may be formed. The c-ABL gene on the long arm of chromosome 9 and the BCR gene on the long arm of chromosome 22 are fused to the $\mathrm{Ph}$ chromosome, forming the BCR-ABL gene. $\mathrm{BCR}-\mathrm{ABL}$ gene-coded $\mathrm{BCR}-\mathrm{ABL}$ thyrosine kinase is produced, and its activation induces neoplastic transformation of cells [21]. In the present case, a chromosome 22 aberration and BCR-ABL gene expression were confirmed by fluorescence in situ hybridization (FISH), thereby verifying that $\mathrm{PN}$ is a tumorous disease.

\section{Conclusion}

We encountered a patient with mandibular INPN and a chromosome 22 aberration. The results of examination using the FISH method demonstrated that INPN is a tumorous disease. This is the first report on BCR-ABL fusion gene expression in the presence of INPN.

\section{Abbreviations}

ENPN: Extraneural perineurioma; FISH: Fluorescent in situ hybridization; INPN: Intraneural perineurioma; PCNA: Proliferative cell nuclear antigen; PCs: Perineurial cells; PN: Perineurioma

\section{Authors' contributions}

JK contributed to the conception and design, acquisition of data, analysis, and interpretation of data. TS and MO participated in the design of the study and performed the statistical analysis. MI contributed to the design and acquisition of data. MS carried out the immunoassays and immunohistochemical staining. SY conceived of the study, participated in its design and coordination, and helped to draft the manuscript. All authors read and approved the final manuscript.

Ethics approval and consent to participate

This study was approved by the ethics committee of Gunma University Graduate School of Medicine.

\section{Consent for publication}

Informed consent was obtained from the patient.

\section{Competing interests}

The authors declare that they have no competing interests.

\section{Publisher's Note}

Springer Nature remains neutral with regard to jurisdictional claims in published maps and institutional affiliations.

\section{Author details}

'Department of Oral and Maxillofacial Surgery, and Plastic Surgery, Gunma University Graduate School of Medicine, 4-39-15 Showa-machi, Maebashi, Gunma 371-8511, Japan. ${ }^{2}$ Department of Pathological Diagnostics, Gunma University Graduate School of Medicine, 4-39-15 Showa-machi, Maebashi, Gunma 371-8511, Japan.

Received: 22 October 2017 Accepted: 21 August 2018

Published online: 13 September 2018

\section{References}

1. Huguet P, De la Torre J, et al. Intraosseous intraneural perineurioma: report of a case with morphological, immunohistochemical and FISH study. Med Oral. 2004:9:64-8.

2. Vencio FE, Cheim PA Jr, et al. Perineurioma of the mandibular dental nerve: a case report and review of the literature. Oral Surg. 2009;2:103-7.

3. Hata $H$, Yamazaki $Y$, et al. Perineurioma of the inferior alveolar nerve with expansion of the mandibular canal. J Oral Maxillofac Surg. 2011;57:419-23.

4. Lazarus SS, Trombetta LD. Ultrastructural identification of benign perineurial cell tumor. Cancer. 1978;41:1823-9.

5. Damm D, White $D$, et al. Intraneural perineurioma-not restricted to major nerves. Oral Surg Oral Med Oral Radiol Endod. 2003;96:192-6.

6. Da Cruz Perez DE, Amanajas de Aguiar FC Jr, et al. Intraneural perineurioma of the tongue: a case report. J Oral Maxillofac Surg. 2006;64:1140-2.

7. Siponen M, Sandor GK, et al. Multiple orofacial intraneural perineuriomas in a patient with hemifacial hyperplasia. Oral Surg Oral Med Oral Pathol Oral Radiol Endod. 2007;104:e38-44.

8. Boyanton $\mathrm{BL}$, Jones JK, et al. Intraneural perineurioma. A systematic review with illustrative cases. Arch Pathol Lab Med. 2007:131:1382-92.

9. Dundr P, Povysil C, et al. Intraneural perineurioma of the oral mucosa. Br J Oral Maxillofac Surg. 2007;45:503-4

10. Kang J, Khang SK, Choi J, et al. Intraneural perineurioma in the tongue. Korean J Pathol. 2007:41:51-4.

11. Tanaka A, Alva PG, Miyazaki Y, et al. Intraneural perineurioma of the tongue: report of a case and review of the literature. Oral Med Pathol. 2009;13:71-4.

12. Rocha LA, SMP L, ARDS S, Lopes MA, Vargas PA. Oral intraneural perineurioma: report of two cases. Clinics. 2009;64:1037-9.

13. Hirano M, Muraki T, Nagayama M, et al. Intraneural perineurioma arising in the lateral border of the tongue. Pathol Int. 2013;63:619-21.

14. Gomes da Silva W, Martinez M, et al. Oral perineurioma: clinicopathologic features from two cases and review of literature. Oral Surg Oral Med Oral Pathol Oral Radiol. 2017;123:e91-8.

15. Emory TS, Scheithauer BW, Hirose T, Wood M, Onofrio BM, Jenkins RB. Intraneural perineurioma: a clonal neoplasm associated with abnormalities of chromosome 22. Am J Clin Pathol. 1995;103:696-704.

16. Giannini C, Scheithauer BW, Jenkins RB, et al. Soft-tissue perineurioma. Evidence for an abnormality of chromosome 22, criteria for diagnosis, and review of the literature. Am J Surg Pathol. 1997;21:164-73. 
17. Barrett AW, Hopper C, et al. Intra-osseous soft tissue perineurioma of the inferior alveolar nerve. Oral Oncol. 2002;38:793-6.

18. Noonan VL, Greene DJ, et al. Extraneural sclerosing perineurioma of the buccal mucosa: a case report and clinicopathologic review. Head Neck Pathol. 2010;4:169-73.

19. Koutlas IG, Scheithauer BW, et al. Intraoral perineurioma, soft tissue type: report of five cases, including 3 intraosseous examples, and review of the literature. Head Neck Pathol. 2010;4:113-20.

20. Koutlas IG, Scheithauer BW. On pseudo-onion bulb intraneural proliferations of the non-major nerves of the oral mucosa. Head Neck Pathol. 2013;7:334-43.

21. Gong Z, Zheng L, et al. Role of complexity of variant Philadelphia chromosome in chronic myeloid leukemia in the era of tyrosine kinase inhibitor therapy. Ann Hematol. 2017:96:501-4.

Ready to submit your research? Choose BMC and benefit from:

- fast, convenient online submission

- thorough peer review by experienced researchers in your field

- rapid publication on acceptance

- support for research data, including large and complex data types

- gold Open Access which fosters wider collaboration and increased citations

- maximum visibility for your research: over $100 \mathrm{M}$ website views per year

At BMC, research is always in progress.

Learn more biomedcentral.com/submissions 